\title{
Station green lighting system based on sensor network
}

\author{
Min Wang ${ }^{1}$, Zhongbo Wu $^{1^{*}}$ and Hang Qin ${ }^{2}$
}

\begin{abstract}
Aiming at the problems of high energy consumption, chaotic lines, and inconsistent lighting sources in many stations, this paper presents a simple, reliable, and convenient station green lighting system based on sensor network. According to the arrival and departure time of the train, the lighting equipment of the corresponding track is automatically closed, which saves the power resources and human resources, and improves the service life of the lighting equipment. In addition, an intelligent illumination control method based on BP neural network is proposed. The test is carried out in the real environment of the station. The designed system can intelligently adjust the working conditions of the lighting system, save energy to the greatest extent and meet the practical requirements.
\end{abstract}

Keywords: Station green lighting system, Sensor network, BP neural network

\section{Introduction}

Railway passenger station is a public place for passengers to get on and off the train. The lighting of its platform, waiting room and other places can provide good visual conditions and comfortable visual environment for passengers and station staff to ensure the service quality of the station and passenger safety. However, at present, there are many shortcomings in station lighting, such as large power consumption, short light source life, backward control mode, and so on [1].

At present, although China's electric power industry is developing rapidly, the situation of insufficient power supply and low power efficiency is still serious, which will continue to exist for a considerable period of time in the future. At the same time, the waste of power resources in the operation and management of stations is quite serious, and the power consumption funds are still high. In practice, the operation and maintenance cost of the station lighting system will even exceed the construction cost, among which the water and electricity cost, management cost, and maintenance cost of mechanical and electrical equipment account for a considerable proportion of the total cost [2].

\footnotetext{
* Correspondence: wuzhongbo@hbuas.edu.cn

${ }^{1}$ Computer Engineering School, Hubei University of Arts and Science,

Xiangyang, China

Full list of author information is available at the end of the article
}

In order to realize the intelligence of a lighting system, some researchers designed an intelligent lighting system based on ZigBee. There are also some intelligent lighting systems based on the Internet of Things architecture. Airports, subway, and other occasions have begun to use intelligent systems to improve lighting efficiency. Chun et al. presents a real-time smart lighting control system using human location estimation [3]. Park et al. put forward a novel model-based PID gain design method to improve the performances of dynamic bending light (DBL) module that change horizontal angle [4]. Some researchers have studied outdoor lighting systems $[5,6]$, while others have studied indoors such as classrooms and homes [7-10]. Wireless control technology was added to the lighting system, but they still need people to participate in and control and have not achieved an energy-saving effect. Some researchers began to use large data and clustering algorithm to analyze the illumination area in order to control the illumination area more intelligently $[11,12]$.

We realize a green lighting system of railway station based on a sensor network. Controlling the lighting equipment automatically according to the arriving and leaving time of the train, the system can economize electric and human resource and improve the using life of the lighting equipment. 


\section{System architecture}

A station green lighting system is mainly composed of a lighting unit, scene controller, and monitoring host, as shown in Fig. 1. The system staff can check, manage, and control the working status of each lighting unit of the whole lighting system by monitoring the host computer. A monitoring host is set up in the system. The host computer is connected to the Internet and installed with the monitoring software of the lighting system. The scene controller and its controlled lighting unit are the basic components of the system. The monitoring host exchange information with the system through the Internet and a GPRS wireless network. The number of scene controllers is determined according to the scale of landscape lighting and application environment. Each scene controller controls 1-127 lighting units. Local communication of the system adopts the ZigBee wireless sensor network, the lighting unit completes the WSN sensor network device function, while the scene controller realizes WSN gateway function and acts as respective sensor network. The coordinator is responsible for the networking and data transmission management of sensor devices. In addition to completing the function of sensor equipment, the lighting unit in the system needs to complete the work including collecting the detection data of the lighting unit, sending data according to the system requirements, battery-charging management, lighting control, and so on.

The automatic control and manual control functions of the lighting system are implemented in the application program of the monitoring server. The main purpose is to control the scene controller according to different conditions, so as to achieve the purpose of controlling the lighting equipment. Its functions include the following:

1. According to illumination, automatic control of lighting equipment is realized. Illuminometers are installed in waiting rooms, train tracks, and squares. Illuminometers are connected with servers. We use $T$ to represent the sample value of the illuminometer, and server-side applications periodically read $T$. For waiting rooms and squares, when $T$ is less than the pre-set threshold $V$ of the program, the corresponding lighting equipment will be closed, when $T$ is greater than $V$, the corresponding lighting equipment will be disconnected; for lanes, when $T$ is less than $V$, the automatic control function of lanes will be activated, and when $T$ is greater than $V$, the automatic control function of lanes will be closed.

2. Controlling scene controllers according to arrival and departure time of trains in the train information table.

The server application program periodically reads the train information in the database. If a train is about to arrive, it opens the lighting equipment of the corresponding controller. If the train on the track leaves, it disconnects the lighting equipment of the corresponding controller.

\section{System implementation}

3.1 Lighting unit

The lighting unit mainly consists of a solar panel, power management module, storage battery, LED lamp control

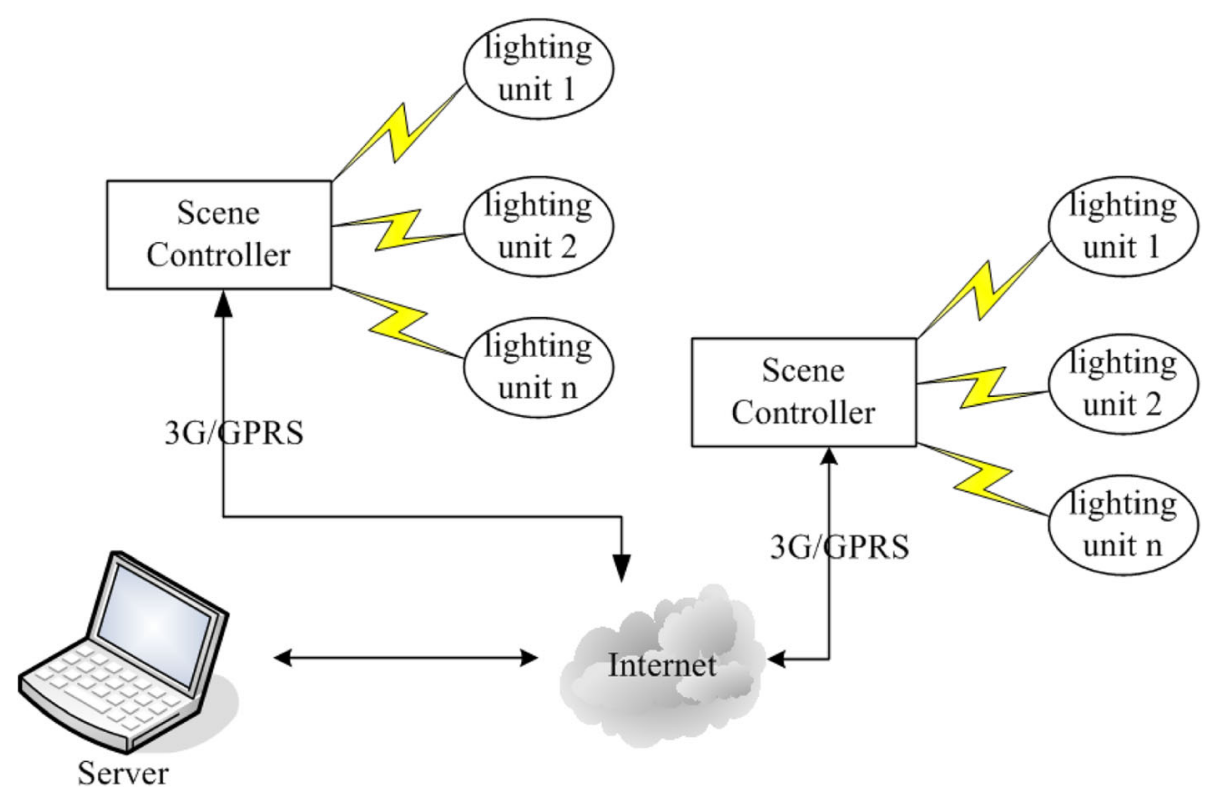

Fig. 1 System architecture 
module, and wireless transceiver module. Solar panels convert light energy into electricity and charge batteries through the power management module. When the lighting system is turned on, the power management module converts the stored energy of the battery into $12 \mathrm{~V} \mathrm{DC}$ required for LED lighting. The power module detects the voltage of the battery in real time. When the voltage of the battery is below the threshold, the module automatically transfers the power of the LED to the market and completes the conversion of $220 \mathrm{VAC}$ to $12 \mathrm{~V} \mathrm{DC}$.

The control module of the LED lamp completes the switch, color adjustment, and dimming of the LED lamp according to the need of the scene setting. At present, LED lamps are usually packaged with $1-\mathrm{W}$ or $3-\mathrm{W}$ beads, which emit different colors of light through different phosphor LED beads. There are three kinds of packaging modes of LED beads: series, parallel, and hybrid. According to the color and brightness requirements of lighting, the packaging mode of LED beads can be selected. Controlling the brightness of LED beads can be achieved by changing the current of the LED beads and adjusting the time of lighting the LED beads. Relatively changing the current adjustment method, using the characteristics of a high flash of the LED to change the lighting time of the LED, is simpler and easier to achieve. It is the main way to adjust the brightness of the LED beads.

\subsection{Monitoring server}

The monitoring server in the system is the information center of the whole lighting system. When the system runs, the upper computer software receives the status information of the lighting unit transmitted from the scene controller via the Internet.

The monitoring server sends queries and setting instructions to the scene controller according to the scene setting requirements, and then forwards them to the corresponding lighting unit by the scene controller.

\subsection{Scene controller}

The scene controller's built-in GPRS module communicates with PC after accessing Internet through GPRS network. At the same time, in the ZigBee wireless sensor network, its role is as a coordinator, responsible for the networking of wireless sensors and the management of sensor equipment. In the system design, the maximum number of communication nodes in each sensor network is set to 128 , that is, one coordinator and $127 \mathrm{de}-$ vices. The number of lighting units in a lighting system may exceed 127 . There are more than two coordinators and their responsible networks in a system at the same time. A unique 16-bit network PANID is set for each coordinator in the system. The embedded ZigBee terminal module in the lighting unit managed by the system needs the same PANID as the network coordinator. In this way, the coordinator located in the scene controller can accept the request to join the network of the same PANID terminal within its network coverage and then add the information of the new lighting unit node.

In the operation of the system, the scene controller does not process and save the information sent by the monitoring host and the lighting unit. It directly sends the state detection information sent by the lighting unit to the monitoring host through the local area network for processing and, at the same time, sends the instructions issued by the monitoring host to the lighting units. The monitoring host is responsible for the information processing and judgment of multiple scenes and lighting units in the whole system. Scene control in the system acts as a sensor gateway, responsible for communication with various devices and Internet network.

The hardware composition of the sensor gateway includes an MCU unit, GPRS module unit, ZigBee module unit, power management unit, and clock unit. The input voltage of the power management unit converts the battery voltage to $4.1 \mathrm{~V}$ required by the GPRS module, $5 \mathrm{~V}$ required by $\mathrm{MCU}$, and $3.3 \mathrm{~V}$ required by the $\mathrm{MCU}$ module. UART0 and UART1 of MCU module are connected with GPRS module and ZigBee module respectively to realize network control and communication. In circuit design, attention should be paid to the fact that the high current at the start of the GPRS module will result in a voltage drop of $0.6-0.7 \mathrm{~V}$. It is necessary to design $1-$ $2100 \mathrm{uF}$ tantalum capacitors between the $4.1-\mathrm{V}$ output terminal and the ground to avoid the restart caused by the protection of GPRS module due to the voltage drop to $3.0 \mathrm{~V}$. The scene controller uses NXPLPC1766 MCU, and its two UART ports are connected with GPRS module and ZigBee transceiver module, respectively. UDP and IP protocol stack are implemented on software based on embedded operating system uC/OS II. The monitoring host in the system can exchange information with a gateway through UDP protocol.

\subsection{Networking process of sensor networks}

The ZigBee wireless sensor network is used for local area communication of lighting control system. It is a low-speed, low-power, and short-distance wireless communication technology. ZigBee supports a variety of networking modes. Considering efficiency and reliability, the system uses star topology networking. Lighting system deploys one or more scene controllers and each scene controller communicates directly with the lighting unit.

The system predefines a PANID for each scenario controller as the network identifier. The scene controller broadcasts broadcast frames $60 \mathrm{~s}$ after starting and opens 
lighting unit's request response to join the network. Once the illumination unit is activated or reset, channel scanning is performed regularly. Once it finds that there are scene controllers available in the network, it sends a request. After the scene controller detects the request, it decides to accept or reject the device to join the network and update its own network table.

In the system, data transmission between scene controller and lighting unit of sensor network adopts direct transmission mode (no intermediate device forwarding), that is, scene control transmits data directly to the lighting unit, and sends confirmation information to the scene controller when the lighting unit receives data. The data transmission mode requires that the terminal node equipment be in the data-receiving state at any time, that is to say, it should be in the wake-up state at any time.

Scene controller uses unicast mode to send information to poll sensor nodes. The scene controller starts up and sends a data transmission request frame to the lighting unit periodically according to the order of lighting units in the network table. The lighting unit receives a request frame, returns a reply frame, and contains its status information in the reply frame.

Scene controller starts to get the IP address and establish a network table. It periodically reports the status information of the illumination unit in the sensor network to the host computer. The default is $5 \mathrm{~min}$, which can be set up. The host computer sets the polling interval of the scene controller through the network and calibrates the local clock of the scene controller. The system adopts a two-level synchronization mechanism to solve the synchronization problem. The upper computer software and the scene controller communication protocol use the checking time frame. The upper computer sends the checking time frame regularly. The scene controller obtains the upper computer time through the frame and checks the local time. In sensor networks, scene controllers send broadcasting pulse frames every $60 \mathrm{~s}$ to synchronize the nodes of the network they manage. The pulse frames contain the updated data of the counter in seconds. After receiving the broadcast pulse frames, the illumination unit updates the counting value of the local timer. The internal timer of the illumination unit updates the counting value of the timer at $1 \mathrm{~s}$. The sensor gateway broadcasts the current time information every $10 \mathrm{~s}$. The sensor gateway contains a clock chip. The internal time counting unit of the sensor network is seconds. The sensor gateway converts the clock chip's $\mathrm{H}: \mathrm{MM}$ :SS into a seconds counting. Each sensor device receives the time data and updates the internal time counter. The timer of each sensor device is interrupted once in $1 \mathrm{~s}$, and the time counter in interruption service is added by 1 .

\subsection{Database design}

The database contains train information table, equipment status table, operation log table, and so on. The most important table is the train information table. The information in this table is the main basis for automatic control of server applications program (Table 1).

The administrator can modify the information of this table through the application program, such as adding, deleting, and modifying the information of the number of trains. Normally, late $=0$, arriveActual $=$ arrival, leaveActual = leave; when the train is late, the administrator modifies the corresponding number of late through the application program. The program automatically modifies arriveActual and leaveActual, arriveActual $=$ arrive + late, and leaveActual $=$ leave + late. When the train leaves, the program automatically modifies late $=0$, arriveActual $=$ arrive, and leaveActual $=$ leave

\section{System improvement}

General lighting for waiting rooms, indoor, and station platforms is generally designed for lighting purposes. Through lighting calculation and scheme design, the illumination of buildings can reach the national lighting standards. But in practice, we all hope that the lighting of various places can not only satisfy people's visual condition of work, but also their study and life. It also hopes to respond to the initiative of national green lighting and save energy consumption (Figs. 2 and 3). Therefore, in the design of the green lighting automatic control system, the first consideration is to combine the illumination and energy saving very well, which can meet its illumination in real time according to its needs, so that the comprehensive index of illumination and energy saving can reach the optimum.

There are some limitations according to the feedback control of illumination. On the one hand, it is difficult to establish an exact mathematical model, and the model is often nonlinear. On the other hand, the open-closed-loop parameter control is more complex which has a large amount of calculation and does not have self-learning

\begin{tabular}{ll} 
Table 1 Database design & \\
\hline Attribute & Description \\
\hline trainid & Train number \\
begin & Departure Station \\
end & Terminus \\
track & Track \\
arrive & Prescribed arrival time \\
leave & Prescribed departure time \\
arriveActual & Actual arrival time \\
leaveActual & Actual departure time \\
late & Delay time \\
\hline
\end{tabular}




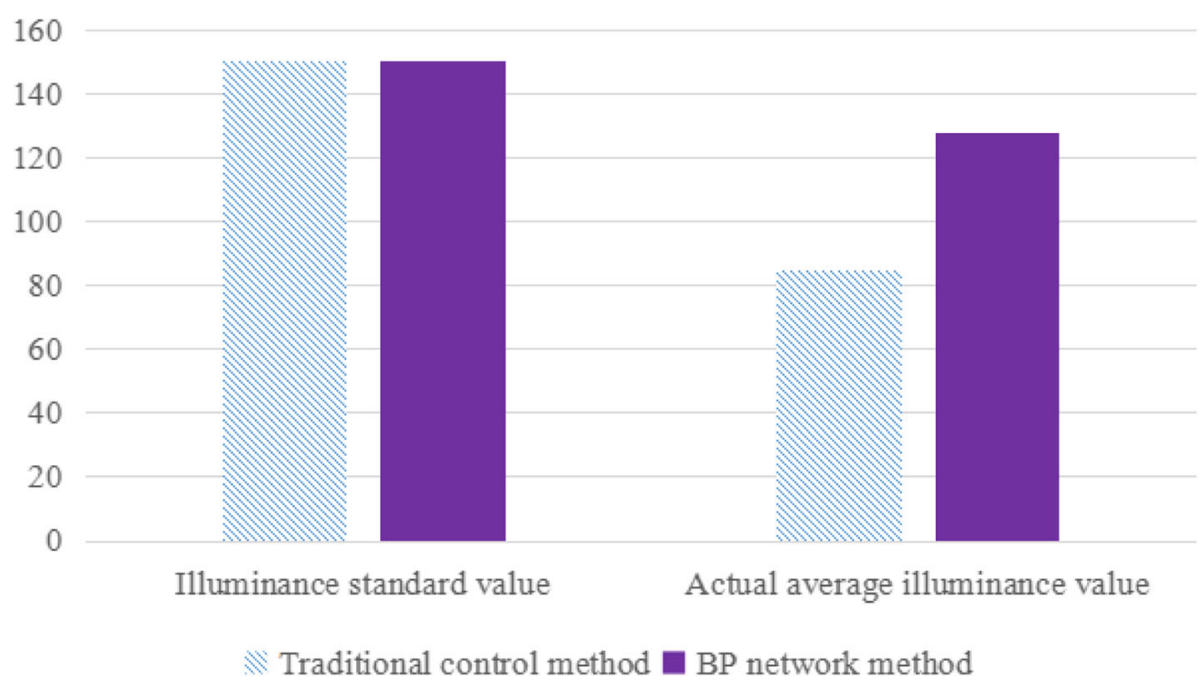

Fig. 2 Comparison of illuminance

function. Real time and accuracy cannot be well guaranteed.

In recent years, artificial neural network (ANN) technology has been developed to the practical stage and has achieved fruitful results in many fields $[13,14]$. A neural network is a large-scale information parallel processing system. It imitates the function of human brain neurons. It has the characteristics of distributed information storage, parallel processing, self-adapting, and self-learning. It can map any function relationship, which is an important function of a neural network.

The illumination control in the green lighting control system can be regarded as a "black box" by combining illumination and power organically according to the variables transmitted by sensors (illumination, voltage, and current). Only the relationship between input and output is studied, and the network is trained by the accumulated experience. In the process of illumination control, as long as the required parameters are provided to the network as input, the intelligent control of illumination can be realized.

BP (back-propagation) network is the most widely used artificial neural network model at present, because its learning algorithm is based on the back-propagation learning algorithm named.

Under certain conditions, there exists a three-layer back-propagation network with input, output, and a nonlinear hidden layer for any given $\varepsilon>0$, which can be used to approximate any nonlinear function with the mean square deviation not exceeding $\varepsilon$.

$P_{1}, P_{2}, P_{3}, \cdots, P_{R}$ is input which can be written in matrix form $P=\left[P_{1}, P_{2}, P_{3}, \cdots, P_{R}\right]$. Their corresponding

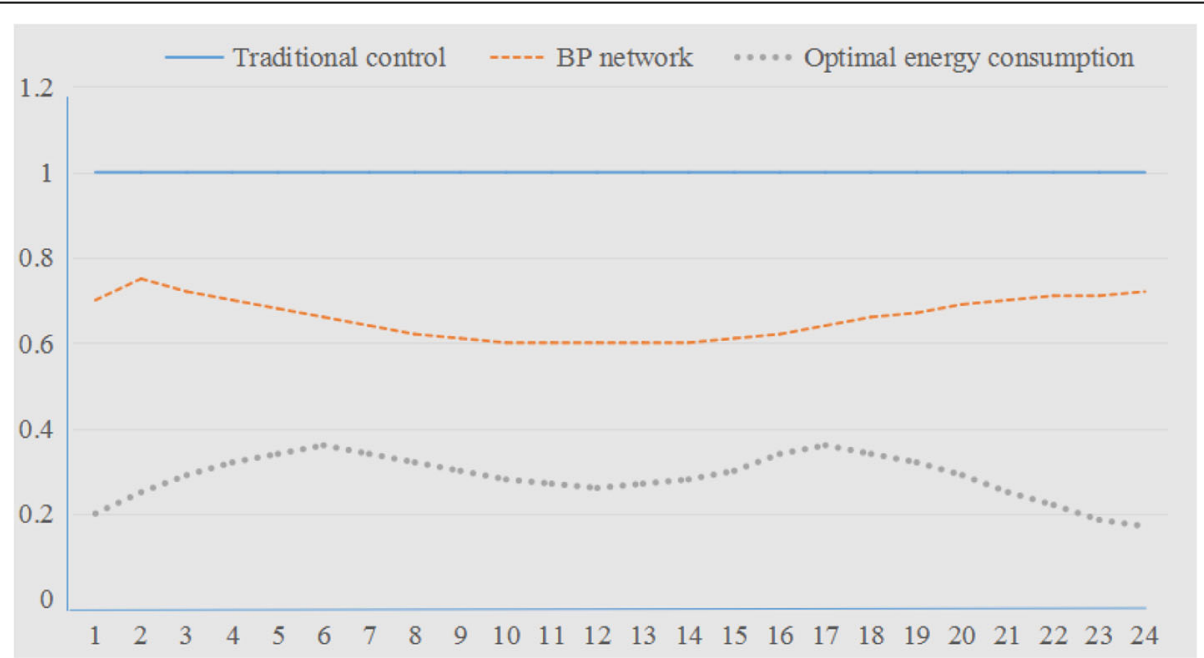

Fig. 3 Comparison of energy consumption 
weights are $W_{1}, W_{2}, W_{3}, \cdots, W_{R}$ which can be written in matrix form $W=\left[W_{1}, W_{2}, W_{3}, \cdots, W_{R}\right]$. Net input of neurons is $n=W_{\mathrm{p}}+b$. Output of neurons is $q=f \cdot W_{\mathrm{p}}+b$.

The weights $W$ and threshold $b$ are all adjustable parameters of neurons, which are adjusted by designers according to learning rules. The input/output relationship is consistent with the design objective. $f$ is a transfer function, which is selected by the designer according to the design objective.

The BP network adopts a supervised learning algorithm. Firstly, we provide a set of input/output data pairs $\left\{x_{1}, t_{1}\right\},\left\{x_{2}, t_{2}\right\},\left\{x_{\mathrm{h}}, t_{\mathrm{h}}\right\}$ as training sample sets. $x_{\mathrm{h}}$ is the input of the network, and $t_{\mathrm{h}}$ is the desired target output response. The training process of the network is to supply each input to the network. The actual output of the network is compared with the target output. The error between the two is regarded as the performance of the network.

We take mean square error as the performance target of BP algorithm.

$$
F(x)=E\left[e^{T} e\right]=E\left[(t-\lambda)^{T}(t-\lambda)\right]
$$

The square error of the $\mathrm{K}$ iteration can be used instead of the upper one.

$$
F=(t(k)-\lambda(k))^{T}(t(k)-\lambda(k))=e^{T}(k) e(k)
$$

Different numerical methods, such as the fastest descent method, Newton method, and conjugate gradient method, can be used to modify the weights and thresholds to reduce errors.

We adopt the fastest descent method.

$$
\begin{aligned}
& W_{i, j}^{m}(k+1)=W_{i, j}^{m}(k)-\lambda \frac{\partial \hat{F}}{\partial W_{i, j}^{m}} \\
& b_{i}^{m}(k+1)=w_{i}^{m}(k)-\lambda \frac{\partial \hat{F}}{\partial w_{i}^{m}}
\end{aligned}
$$

$\lambda$ is the learning rate $\frac{\partial \hat{F}}{\partial W_{i, j}^{m}}=\frac{\partial \hat{F}}{\partial n_{i}^{w}} \times \frac{\partial n_{i}^{m}}{\partial W_{i, j}^{m}}$

$$
\frac{\partial \hat{F}}{\partial b_{i}^{m}}=\frac{\partial \hat{F}}{\partial n_{i}^{w}} \times \frac{\partial n_{i}^{m}}{\partial b_{i}^{m}}
$$

$n_{i}^{m}$ is the net input of layer $m n_{i}^{m}=\sum_{j-1}^{T^{m-1}} W_{i, j}^{m} \lambda_{i}^{m-1}+b_{i}^{m}$

So $\frac{\partial \eta_{i}^{m}}{\partial W_{i, j}^{m}}=\lambda_{j}^{m-1}, \frac{\partial n_{i}^{m}}{\partial b_{i}^{m}}=1$.

Definition sensitivity $C_{i}^{m}=\frac{\partial \hat{F}}{\partial n_{i}^{m}}$ (That is the change rate of the $i$ th element to the net input of $m$ level by $\hat{F}$.)

$$
\frac{\partial \hat{F}}{\partial W_{i, j}^{m}}=T_{i}^{m} \alpha_{j}^{m-1}, \frac{\partial \hat{F}}{\partial b_{i}^{m}}=C_{i}^{m}
$$

Now the fastest descent method can be written as

$$
W_{i, j}^{m}(k+1)=W_{i, j}^{m}(k)-\lambda C_{i}^{m} \lambda_{j}^{m-1}, b_{i}^{m}(k+1)=b_{i}^{m}(k)-\lambda C_{i}^{m}
$$

Its matrix form can be written as

$$
\begin{gathered}
W^{m}(k+1)=W^{m}(k)-\lambda C^{m}\left(\lambda^{m-1}\right)^{T}, b^{m}(k+1)=b^{m}(k)-\lambda C^{m} \\
C^{m}=\frac{\partial \hat{F}}{\partial n^{m}}=\left[\begin{array}{l}
\frac{\partial \hat{F}}{\partial n_{1}^{m}} \\
\frac{\partial \hat{F}}{\partial n_{2}^{m}} \\
\cdots \hat{F} \\
\frac{\partial \hat{F}}{\partial n_{n}^{m}}
\end{array}\right]
\end{gathered}
$$

Sensitivity should be calculated from the last formula to the first layer by the recursive formula.

$$
C^{m} \rightarrow C^{m-1} \cdots C^{2} \rightarrow C^{1}, C^{m}=\left(\frac{\partial n^{m+1}}{\partial n^{m}}\right)^{T} \frac{\partial \hat{F}}{\partial n^{m+1}}
$$

We use the Jacobi matrix to derive the recurrence relation of sensitivity.

$$
\frac{\partial n^{m+1}}{\partial n^{m}}=\left[\begin{array}{cccc}
\frac{\partial n_{1}^{m+1}}{\partial n_{1}^{m}} & \frac{\partial n_{1}^{m+1}}{\partial n_{2}^{m}} & & \frac{\partial n_{1}^{m+1}}{\partial n_{s}^{m}} \\
\frac{\partial n_{2}^{m+1}}{\partial n_{1}^{m}} & \frac{\partial n_{2}^{m+1}}{\partial n_{2}^{m}} & \cdots & \frac{\partial n_{2}^{m+1}}{\partial n_{s}^{m}} \\
\frac{\partial n_{s}^{m+1}}{\partial n_{1}^{m}} & \frac{\partial n_{s}^{m+1}}{\partial n_{2}^{m}} & & \frac{\partial n_{s}^{m+1}}{\partial n_{s}^{m}}
\end{array}\right]
$$

The elements $(i, j)$ in the matrix can be calculated by the following formula.

$$
\begin{aligned}
\frac{\partial n_{i}^{m+1}}{\partial n_{j}^{m}} & =\frac{\partial\left[\sum_{i-1}^{C^{m}} W_{i, j}^{m+1} \lambda_{j}^{m}+b_{i}^{m+1}\right]}{\partial n_{j}^{m}} \\
& =W_{i, j}^{m+1} \frac{\partial \lambda_{j}^{m}}{\partial n_{j}^{m}}=W_{i, j}^{m+1} \frac{\partial f^{m}\left(n_{j}^{m}\right)}{\partial n_{j}^{m}} \\
& =W_{i, j}^{m+1} f^{m}\left(n_{j}^{m}\right)=W^{m+1} F^{m}\left(n^{m}\right)
\end{aligned}
$$

The sensitivity formula can be rewritten as $C_{j}^{m}=-2($ $\left.t_{j}-\lambda_{j}\right) f^{m}\left(n_{j}^{m}\right)$.

\section{Experiments}

\subsection{Simulation setup}

The neural network selects a three-layer BP network with a hidden layer. The input and output of the 
Table 2 Eight neurons

\begin{tabular}{ll}
\hline Symbol & Definition \\
\hline$u$ & The actual illuminance value measured by sensors \\
$i$ & Voltage measured by sensor \\
$E$ & Current measured by sensor \\
$O$ & Illuminance standard value \\
$L$ & Choosing luminous flux of light source \\
$W$ & The length of the platform \\
$H$ & The width of the platform \\
\hline
\end{tabular}

network depend on the definition of the external problem. For the lighting system which needs to adjust the illumination on-line in real time, in order to satisfy the illumination conditions and achieve the optimal energy saving, even if the comprehensive index of illumination and power reaches the optimum, the input layer selection of the network depends on the definition of the external problem. The eight neurons are as follows (Table 2).

With each group of lights as the output (there are eight groups of lights here), according to the value of illumination, automatically realize the switch of each group of lights, so that the illumination remains at a predetermined value, so that only eight neurons in the output layer, the number of neurons in the hidden layer using the gradual growth method to determine, that is, starting with a simple network, if it does not meet the requirements, gradually increase the number of neurons in the hidden layer until appropriate.

\subsection{Results and discussion}

With the intelligent lighting control system, the lighting system can work in the full automatic state system and automatically adjust the illumination to the most suitable level according to the preset value. This can make good use of natural light, and when the weather or season changes, the system can ensure that the light in the waiting room is maintained at a predetermined level. From the simulation results, it can be seen that the BP network algorithm in the neural network can not only make the illumination satisfactory and make the fatigued passengers be in a comfortable environment, but also save considerable energy. It can save $25-45 \%$ of the energy, which is in line with the concept of green lighting.

Figure 4 shows our green lighting system, which turns on the traffic lights of the corresponding lanes $5 \mathrm{~min}$ before the train arrives and turns off the traffic lights of the corresponding platforms $10 \mathrm{~min}$ after the train leaves. When you click the manual control button, an open and closed control panel will be displayed on the interface. You can click on or off the button to control the corresponding track lights. The system is widely used in Xiangyang, Luoyang, Sanmenxia, and other stations, and each station can save $30-50 \%$ of the electricity cost each year.

\section{Conclusion}

This paper puts forward the concept of "green lighting" for station lighting, which not only has great significance for saving electric energy and providing a healthy and comfortable environment for passengers, but also promotes the implementation of a green lighting project in China. In the automatic control system of green lighting, the intelligent control of illumination based on neural network theory is proposed, which has certain theoretical research significance and practical application value.

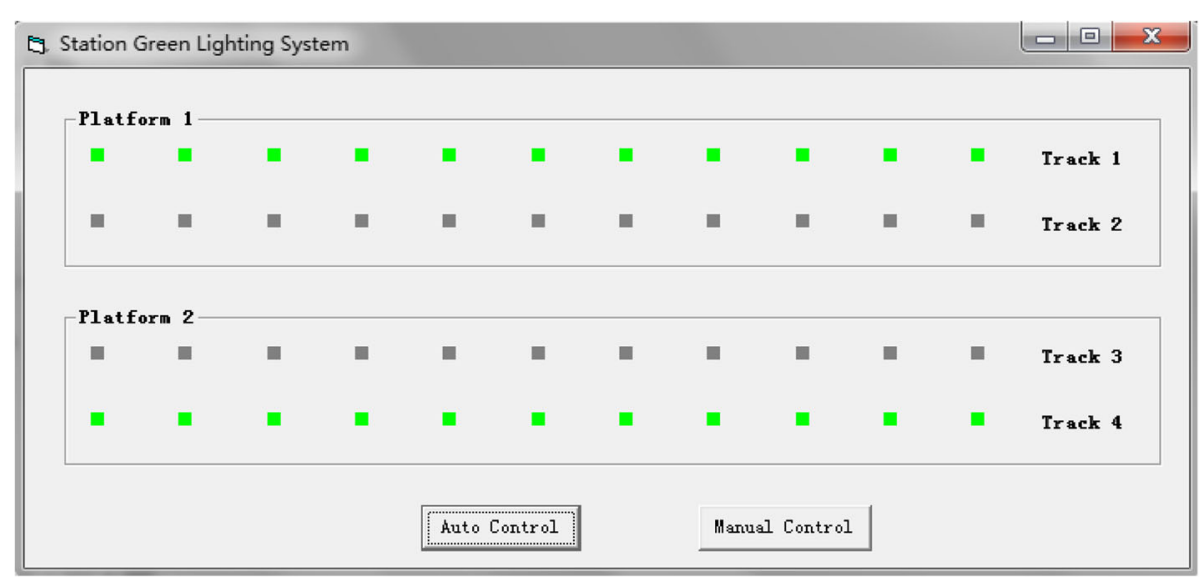

Fig. 4 Interface of actual system 


\section{Funding}

The authors acknowledge the Hubei Ministry of Education Foundation (Grant: B2016176) and Open Fund of Hubei Superior and Distinctive Discipline Group of "Mechatronics and Automobiles".

\section{Availability of data and materials}

Data sharing not applicable to this article as no datasets were generated or analyzed during the current study.

\section{Authors' contributions}

MW carried out the design of system architecture and drafted the manuscript ZW carried out the development of prototype system. HQ participated in the design of the study and performed the statistical analysis. ZW conceived of the study, participated in its design and coordination, and helped to draft the manuscript. All authors read and approved the final manuscript.

\section{Competing interests}

The authors declare that they have no competing interests.

\section{Publisher's Note}

Springer Nature remains neutral with regard to jurisdictional claims in published maps and institutional affiliations.

\section{Author details}

${ }^{1}$ Computer Engineering School, Hubei University of Arts and Science, Xiangyang, China. ${ }^{2}$ Computer School, Yangtze University, Jingzhou, China.

Received: 18 February 2019 Accepted: 10 April 2019

Published online: 29 April 2019

\section{References}

1. R. Fiederling, "Smartrix" a new chapter in the smart matrix LED lighting. ATZelektronik Worldw. 12(4), 50-55 (2017)

2. X. Ye, X. Xia, Optimal lighting project maintenance planning by a control system approach. IFAC Proc. Vol. 47(3), 3152-3157 (2014)

3. S.Y. Chun, C. Lee, J. Jang, et al., Real-time smart lighting control using human motion tracking from depth camera. J. Real-Time Image Proc. 10(4), 805-820 (2015)

4. S.H. Park, B.U. Im, D.K. Park, et al., Model based optimum Pid gain design of adaptive front lighting system. Int. J. Automot. Technol. 19(5), 923-933 (2018)

5. I. Wojnicki, S. Ernst, L. Kotulski, et al., Economic impact of intelligent dynamic control in urban outdoor lighting. Energies 9(5), 1-14 (2016)

6. L. Tahkamo, R.S. Rasanen, L. Halonen, et al., Life cycle cost comparison of high-pressure sodium and light-emitting diode luminaires in street lighting. Int. J. Life Cycle Assess. 21(2), 137-145 (2016)

7. H. Lee, S. Kwon, J. Lim, et al., A development of a lighting control system based on context-awareness for the improvement of learning efficiency in classroom. Wirel. Pers. Commun. 86(1), 165-181 (2016)

8. M.K. Jha, N. Kumar, Energy efficient lighting system for indoor parking with ubiquitous communication. Wirel. Pers. Commun. 100(2), 379-389 (2018)

9. A. Yano, K. Fujiwara, Plant lighting system with five wavelength-band lightemitting diodes providing photon flux density and mixing ratio control. Plant Methods 8(1), 46-46 (2012)

10. S. Moon, S. Kwon, J. Lim, et al., Implementation of smartphone-based color temperature and wavelength control LED lighting system. Clust. Comput. 19(2), 949-966 (2016)

11. F. He, L. Zhang, Mold breakout prediction in slab continuous casting based on combined method of GA-BP neural network and logic rules. Int. J. Adv. Manuf. Technol. 95(95), 4081-4089 (2018)

12. C. Zhang, C. Liu, X. Zhang, et al., An up-to-date comparison of state-of-theart classification algorithms. Expert Syst. Appl. 82(82), 128-150 (2017)

13. A.H. Moghaddam, M.H. Moghaddam, M. Esfandyari, et al., Stock market index prediction using artificial neural network. J. Econ. Finance Adm. Sci. 21(41), 89-93 (2016)

14. H. Zhenlong, Z. Qiang, W. Jun, et al., The prediction model of cotton yarn intensity based on the CNN-BP neural network. Wirel. Pers. Commun. 102(2), 1905-1916 (2018)

\section{Submit your manuscript to a SpringerOpen ${ }^{\circ}$ journal and benefit from:}

- Convenient online submission

- Rigorous peer review

- Open access: articles freely available online

High visibility within the field

- Retaining the copyright to your article

Submit your next manuscript at $\boldsymbol{\nabla}$ springeropen.com 